\title{
A review of COVID-19: A summary of the epidemic in Wuhan and other local areas in China
}

\author{
Kaiyin $\mathrm{Hu}^{1, *, \dagger}$, Yichao $\mathrm{Su}^{2, \dagger}$, Jiaqi Wang ${ }^{3, \dagger}$, Yuzhe $\mathrm{Xu}^{4, \uparrow}$ \\ ${ }^{1}$ College of Education, Health and Human Sciences, University of Tennessee, Knoxville, 37996, Knoxville, The United States \\ ${ }^{2}$ School of Nursing, Sun Yat-sen University, 510000, Guangzhou, China \\ ${ }^{3}$ College of Integrated Chinese and Western Medicine, Tianjin University of Traditional Chinese Medicine, 301617, Tianjin, China \\ ${ }^{4}$ School of Public Health (Shenzhen), Sun Yat-sen University, 510000, Guangzhou, China \\ ${ }^{\dagger}$ These authors contributed equally.
}

\begin{abstract}
COVID-19 is a new kind of coronavirus that was found in 2019 and it has swept the world until now. Reviewing it and discovering the problem is what we must do. In this scenario, the influence of COVID19 on the world, and especially in China since it is the country where COVID-19 first outbreak, has been thoroughly reviewed. The main point of this paper is to identify the epidemiological information of COVID19 and to review typical cities that are most influenced by COVID-19, such as Wuhan, Dalian, and Shijiazhuang. Moreover, discovering and comparing the characteristics and regulations according to different periods have also been carefully performed. With the understanding of the infection of COVID-19, the bright future in which prevention and control measures of nosocomial infection are improved is surely within reach. Furthermore, the psychological construction and management in fundamental systems require more attention.
\end{abstract}

\section{Introduction}

COVID-19 is a new coronavirus found in 2019, and it influences people's respiratory system and causes by SARS-CoV-2. This virus has powerful infections, and it is thought to spread from one individual to another through the respiratory droplets caused when an infected patient coughs or talks.

It is significant to study COVID-19 because this virus influences a country and the whole world. This virus brought a big smash to the world's medical system, which caused the collapse of the medical system and a lot of people were dead because of COVID-19. COVID-19 also influenced people's lives, and people need to constantly aware of this virus. Studying this virus becomes necessary because it helps scientists know more about this COVID19 and help them make vaccine or medicine for this disease.

According to the current situation in China, the Chinese government has controlled the deterioration and development of the epidemic. Basically, all cities have returned to the pace and quality of life before the epidemic happen, and people have begun to receive vaccines in prescribed medical institutions. Nowadays, People do not have to wear masks, except when entering some public places, such as malls, public transportation, schools. However, the control of the epidemic is not absolute, and there will still be sudden epidemics in some cases. According to the situation in overseas, the COVID-19 still influence people's life negatively. The number of cases is still increasing. Even people gradually begin to accept the vaccine, but still, many people do not want to take it. Thus, COVID-19 still is a big problem to the world.

To have a more comprehensive understanding of the COVID-19 outbreak in China, we present a review of the COVID-19 epidemic, prevention, control, and treatment in China, as well as the transmission, prevention, control, the relevant policies and solutions of the government in some representative areas. The primary organization of this paper is divided into three main parts. In Part 1 of the main body, we describe the general COVID-19 epidemic and the current situation of outbreak control in China, as well as the treatment. In Part 2 of the main body, our analysis of the epidemic situation in China is refined to four representative cities (Wuhan, Dalian, Shenyang, and Shijiazhuang) representing four different modes of outbreak and transmission. In Part 3 of the main body, by comparing the four cities mentioned in the second part, we collect the policies and solutions proposed by the local government. The implementation and effectiveness of these policies are discussed.

\section{COVID-19}

The novel coronavirus pneumonia epidemic on January 30, 2020, was identified by World Health Organization (WHO) as a Public Health Emergency of International Concern (PHEIC), and the post pathogenic virus was officially named as 2019 New Coronavirus (February 11, 2020 ) on January 30, 2020 [1]. It has been proved that it belongs to coronavirus $\beta$ [2], which is $80.3 \%$ similar to the human SARS virus [3]. It has been included in class B

${ }^{*}$ Corresponding author: khu3@vols.utk.edu 
infectious disease in China in early 2020 and managed as a class A infectious disease [4]. Clinical evidence suggests that New Coronavirus's host may be a bat that spreads to humans through certain animal hosts and causes epidemics. At present, the main source of infection is New Coronavirus infected patients, and the latent infection may also become the source of infection, mainly through respiratory droplet transmission and contact transmission, and the crowd is generally susceptible. The average incubation period was 5.2 days, and the number of regenerations was 2.2 in the early stage of the epidemic. Most of the patients presented as normal type and light type. The mortality rate was $2.38 \%$. The mortality rate of elderly men with underlying diseases was higher $[5,6]$. This time, the new coronavirus pneumonia has a strong infectious ability and needs to be well prepared by all mankind.

\subsection{Epidemic situation of COVID-19}

As of October 9, 2020, 91263 people have been diagnosed and 4746 people have died in China, with a fatality rate of $5.20 \%$; overseas 36729097 people have been diagnosed and 1063102 people have died, with a fatality rate of $2.89 \%$; as of January 27, 2021, 100499 people have been diagnosed and 4818 people have died in China, with a fatality rate of $4.80 \%$; overseas 101009 people have been diagnosed, 117 people, a total of 2167216 people died, the fatality rate was $2.15 \%$ [7]. It can be considered that although the epidemic situation in China has rebounded from October 2020 to now, it has been controlled overall, while the overseas epidemic situation is still spreading rapidly, but the mortality of both has been reduced, which may be that the anti-epidemic measures have played a certain effect.

According to the novel coronavirus pneumonia, the new economic situation in Johns Hopkins University has been reported again on August 2nd. As of July 31, about $70 \%$ (126) countries and regions in the world have increased the number of newly diagnosed cases. China's Beijing, Jilin, Xinjiang, and other places also rebounded. China's National Health Commission announced on September 21, 2020, that 12 new confirmed cases were imported from 31 provinces on September 20, which was also the zero new local cases in mainland China for 36 consecutive days. As of December 7, 2020, there have been confirmed cases in Sichuan, Shanghai, Zhejiang, and other places in mainland China. Therefore, although the epidemic situation in mainland China has been controlled temporarily, there is still a great risk of rebound

\subsection{Treatment}

The treatment principle of all infectious diseases is to control the source of infection and block transmission. The novel coronavirus pneumonia is also an exception. novel coronavirus. Pneumonia patients are the main source of infection, isolation of patients is particularly important. Dividing patients is not only a measure to control the source of infection but also a way to block transmission.
The clinical classification of 2019-COVID can be divided into four types: light, general, heavy, and dangerous in China. Mild and common types belong to mild, severe, and critical types belong to severe. Mild patients with mild clinical manifestations, or with fever, respiratory symptoms, imaging visible ground glass-like performance, no dyspnea, chest tightness performance. Most severe patients have shortness of breath, respiratory rate is more than 30 times/ min, oxygen saturation is less than $93 \%$, there is hypoxemia, severe cases can appear respiratory failure, shock, imaging manifestations 1-2 days, the lesion progress is more than 50\%, need respiratory support, there are other organ failures, need support treatment [8].

Mild patients: Based on syndrome differentiation, comprehensive use of traditional Chinese medicine decoction, traditional Chinese medicine, proprietary medicine, TCM nursing appropriate technology, Baduanjin, and other characteristic therapies. Point hospital set up integrated traditional Chinese and Western medicine ward, Chinese medicine expert group [9].

In the process of treatment, we should pay attention to the patient's chief complaint, timely review the blood routine, blood gas analysis and other indicators, as well as chest X-ray, and be alert to the deterioration of mild patients' lung conditions and pay attention to the occurrence of complications, discharge standards should be strictly in accordance with the provisions, to prevent the phenomenon of re positive [10].

Severe patients: Although novel coronavirus pneumonia patients are novel coronavirus pneumonia patients, the proportion of all cases is not high, but severe pneumonia is very dangerous.

Therefore, we need to give full play to the advantages of modern medical monitoring technology and organ function support technology, apply traditional Chinese medicine treatment based on simple conventional western medicine treatment, to achieve better clinical efficacy, maximize the use of integrated traditional Chinese and Western medicine, and protect people's lives and health. Chloroquine phosphate has been recommended for antiviral treatment of new coronavirus [11].

Cytokine storm novel coronavirus pneumonia is a major cause of death in patients with new crown pneumonia, for patients with extensive bilateral lung disease and severe lung disease, and the level of IL- 6 detected in the laboratory is increased, it is recommended to try tobuzumab treatment [12].

\section{Epidemic outbreak revisions}

\subsection{Pandemic in Wuhan}

\subsection{1 overview}




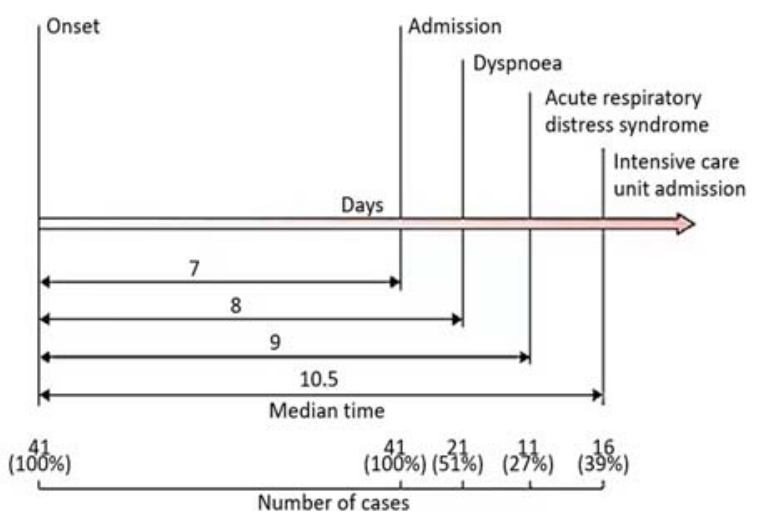

Figure 1. Timeline of 2019-nCoV cases after the onset of illness [13].

The pandemic was caused by a novel beta-coronavirus, the 2019 novel coronavirus (2019-nCoV) outbroke in Wuhan in December 2019. Wuhan city was attacked by the virus and closed firstly in China. This kind of coronavirus was highly contagious and infectious, plenty of people were infected in a short period. 41 patients had been identified as having laboratory-confirmed 2019nCoV infection till January 2nd, 2020 (Fig.1) [13].

On February 12th, 2020, there were 14840 new cases confirmed, which was the highest number of confirmed cases in a single day over the course of the epidemic. Then from February 12th to May 6th, the number of COVID19 infections continued to decline, until May 6th, 2020, when the number of infections reached zero. Since then, the number of new cases in Hubei province has been zero (except for import cases) (Fig.2) [14].

\subsubsection{The source of the epidemic}

On December 31, 2019, the Wuhan Health Commission announced that certain unidentified pneumonia that recently appeared in Wuhan was all related to the South China Seafood Market. After receiving the report, the Municipal Health Commission carried out a retrospective investigation immediately and searched the cases related to South China Seafood Market in the city's medical and health institutions. As of December 31, 27 cases have been found. 7 cases were in serious condition, the rest of them were stable and controllable ( 2 cases are expected to be discharged from the hospital soon). The main clinical manifestations of the cases were fever, a few patients with dyspnea, a chest radiograph showed bilateral lung infiltration lesions [15].
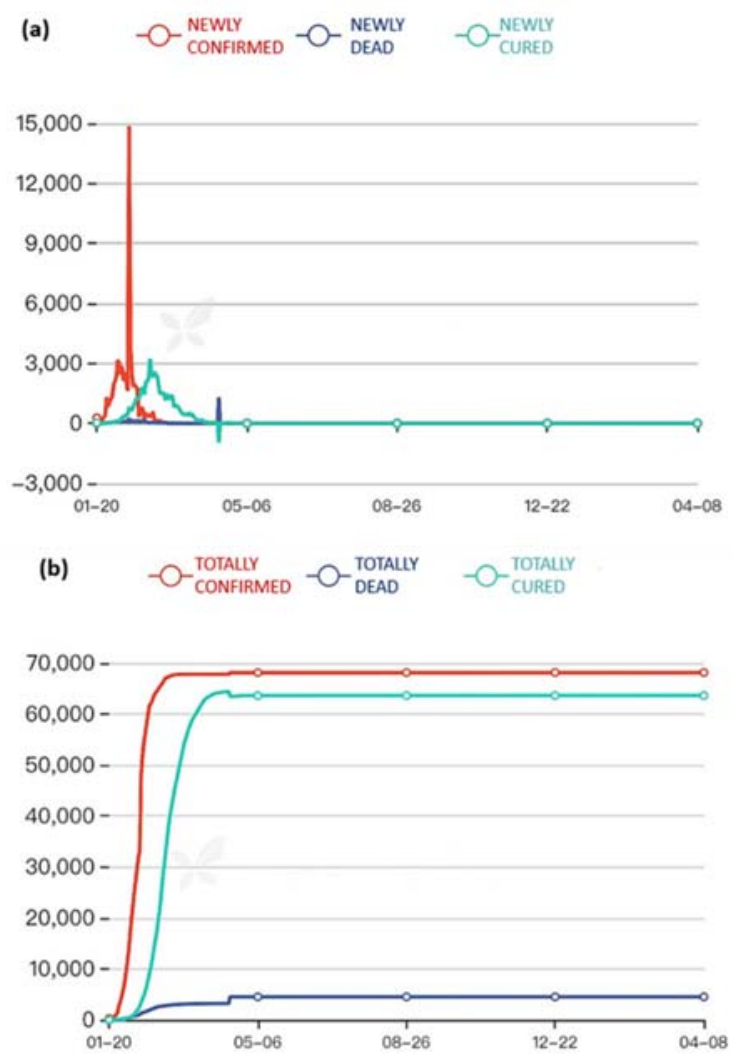

Figure 2. (a) New confirmed and recovered cases in China and Hubei province; (b) Number of people affected, recovered, and dead in China and Hubei province [14].

\subsubsection{Virus features}

$\mathrm{Xu}$ et al. performed phylogenetic analysis on the collection of coronavirus sequences from various sources. The results showed the Wuhan CoVs were clustered together in the phylogenetic tree, which be- long to the Beta coronavirus genera (Fig. 3). The S-protein of coronavirus is divided into two functional units, S1 and $\mathrm{S} 2$. S1 facilitates virus infection by binding to host receptors. It comprises two domains, the N-terminal domain and the C-terminal RBD domain that directly inter- acts with host receptors (Li, 2012). To investigate the Wuhan $\mathrm{CoV}$ and its host interaction, $\mathrm{Xu}$ et al investigated the RBD domain of its S-protein. The Sprotein was known to usually have the most variable amino acid sequences compared to those of ORF1a and ORF1b from coronavirus (Hu et al., 2017) [16].

\subsubsection{Route of transmission}

There were four major transmission routes identified in the pandemic [17]:

a. Droplet and contact transmission.

b. Aerosol transmission [18];

c. Fecal-oral transmission.

d. Mother-to-child transmission (PMTCT). 


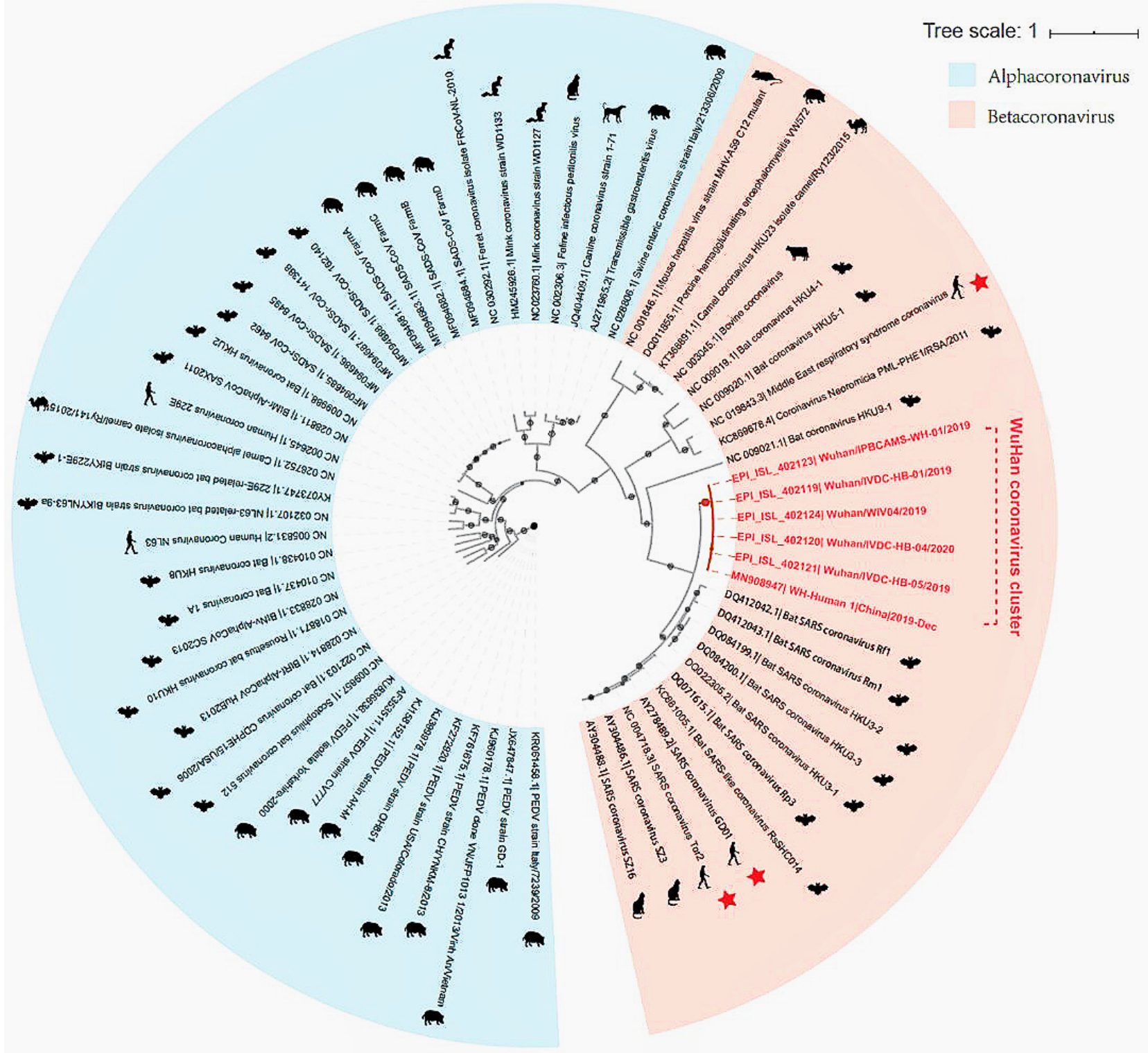

Figure 3. Evolutionary analysis of the coronaviruses and modeling of the Wuhan CoV S-protein interacting with human ACE2. A Phylogenetic tree of coronaviruses based on full-length genome sequences. The tree was constructed with the Maximum-likelihood method using RAxML with GTRGAMMA as the nucleotide substitution model and 1,000 bootstrap replicates. Only bootstraps $\geq 50 \%$ values are shown as filled circles. The host for each coronavirus is marked with a corresponding silhouette. Known humaninfecting beta-coronaviruses are indicated with a red star [16].

\subsection{Pandemic in Dalian}

\subsubsection{Overview}

In December 2020, the COVID-19 was largely under control. Dalian, China's northernmost port city had seen another outbreak due to importing goods. Operations at the port were briefly interrupted and according to some reports, the cold chain portion of the port remains closed. Chinese state media reported that the outbreak was identified on December 15 with an asymptomatic individual who reportedly worked as a loader for a cold chain importer. Other reports mentioned a restaurant worker. The Chinese media said that testing began and operations at the cold-chain supply were paused to ensure that no contaminated food entered the supply chain [19].

\subsubsection{The epidemic developments}

From December 15, 2020, to 24, the outbreak grew with between 15 and 20 confirmed patients and as many as 20 additionals confirmed asymptomatic cases. The government announced that they had added seven patients on Thursday, December 24 as well as one more positive test on a person not showing symptoms, China increased its response across the city [19]. 
a. Dockworkers and subsequent transmission of the epidemic (10 cases);

b.Spreading from cold chain terminals to communities, shopping malls, schools, hospitals, and all kinds of people (Internal communication,65 cases) [20].

\subsection{Pandemic in Shenyang}

\subsubsection{Overview}

A case of novel coronavirus positive nucleic acid test after a South Korean returned to Shenyang was released from quarantine. Yin XX, a female, 67 years old, Chinese national, arrived at Shenyang Taoxian Airport on November 29, 2020, on a CZ682 flight from South Korea. Shenyang Customs reported that her first nucleic acid test result was negative. According to the control measures for foreign personnel entering Shenyang, they were immediately transferred point-to-point by special buses to the designated observation point for foreign personnel entering Shenyang for centralized quarantine for medical observation for 14 days. During the centralized quarantine, nucleic acid and serum antibody test results were negative. The quarantine was lifted on 13 December.

At 20:00 on December 22, 2020, the patient went to the fever clinic of the First Affiliated Hospital of China Medical University. At 2:40 on December 23, the hospital diagnosed the patient as a suspected case. Municipal Center for Disease Control and Prevention collected the biological specimens for the nucleic acid test, the results were positive. Shenyang Municipal Commission of Health and Health immediately started the transfer procedure and transferred the patient to the Sixth People's Hospital for diagnosis and treatment [21].

\subsubsection{The epidemic developments}

There were no new confirmed cases of COVID-19 and no new asymptomatic infections in Liaoning Province from $0: 00$ to $12: 00$ on January 16,2021 . One new local confirmed case was cured and discharged from the hospital, which was reported in Shenyang City.

As of 12 o 'clock on January 162021,396 confirmed cases (including 85 imported cases) have been reported in the province, of which 359 have been cured and discharged, 2 have died and 35 have been treated in hospital. At present, there are 12 asymptomatic infected people in designated hospitals in isolation treatment [22].

\subsection{Pandemic in Shijiazhuang}

\subsubsection{Overview}

On January 2, 2020, the first infection case was detected in Xiaoguozhuang Village, Gaocheng District, Shijiazhuang City, Hebei Province. The next day, authorities carried out a nationwide nucleic acid test in Xiaoguzhuang and nearby villages. Gradually the outbreak expanded the screening scope. As of January 10, 2021, Shijiazhuang had completed the first round of nucleic acid tests for 10.25 million people, with a total of 249 confirmed infections, more than 85 percent of which were in Gaocheng [23].

\subsubsection{Transmission characteristics}

According to the Shijiazhuang Center for Disease Control and Prevention, the epidemic was mainly concentrated in a high spatial pattern, and the infected people were mainly in rural areas. This reflects the weakness of rural epidemic prevention and control capacity. The grass-roots epidemic prevention front, accustomed to "passive epidemic prevention", was vulnerable to a sudden virus, which roamed freely on the back of a tight network of acquaintances in the village [23].

\section{Characteristic, regulation, and referential from different periods of pandemic}

In the first month of the 2020s, the Wuhan government discovered about 44 cases with unexplained viral pneumonia diagnoses, and 11 cases were serious [24]. According to the epidemiology, the investigation shows that most cases were from South China Seafood Market in Wuhan. At that time, there was no obvious evidence to prove that human-to-human transmission existed. However, after one month, the situation turns to a different level.

Data collected from November 2019 to January 2020. It presents the earliest cases that included reported exposure to the Huanan Seafood Market. Still, there was a significant increase in late December, and those cases were not linked with the Seafood market [25].

As of February 1st, 2021, Hubei province (where the Wuhan is) had confirmed 1921 cases; 268 new severe cases in Hubei Province, 45 new deaths, and 49 new cured and discharged cases were recorded from Hubei Province. There were 2606 new suspected cases were detected in Hubei Province [24]. According to the data, the spread of the COVID-19 has started quickly, which shows the situation in Wuhan was catastrophic.

Since the Wuhan Government did not immediately arouse the virus's attention and did not publish any measures, it led to the rapid spread of COVID-19 among the people. It became serious and gradually caused people anxiety. The outbreak of the epidemic also influenced the hospital. It caused a serious shortage of medical resources and threatened the health and safety of medical staff. Simultaneously, the Chinese government had already paid attention to the epidemic that happened in Wuhan. On January 15th, 2020, On January 15th, 2020, Hubei Province requested Tongji Hospital, Provincial People's Hospital, and Union Hospital to take over the South Seventh Floor, South Sixth Floor, and South Fifth Floor of Jinyintan Hospital, respectively, and all these three floors were changed to ICU wards, and at the same time, the municipal hospitals deployed staff to support them. On the same day, the Chinese Center for Disease Control and Prevention launched a first-level emergency response. On January 18th, Zhong Nanshan, a Chinese pulmonologist 
and famed for managing the SARS outbreak, led a team of experts to Wuhan because of the Chinese government's request. It was starting on January 23rd. Wuhan began to "lockdown" [26].

\subsection{Lockdown in Wuhan}

According to Wuhan's situation, the Chinese government decided to lock down the city after a long discussion with experts. The decision was kind of inflexible, but it was correct and necessary. Wuhan's lockdown earns the time for China because it slowed the spread of the epidemic to other cities by 2.91 days, thus delaying the transmission of the disease in other cities [27]. During the lockdown period, people were not allowed to leave or going to Wuhan. All the public transport in Wuhan was suspended, such as bus and subway; school and entertainment have been closed. All the public gatherings were prohibited [27], and the measures of stopping public transportation had an influential association with a decrease in case incidence [27]. However, what if the lockdown and any other measures were not implemented? Based on the

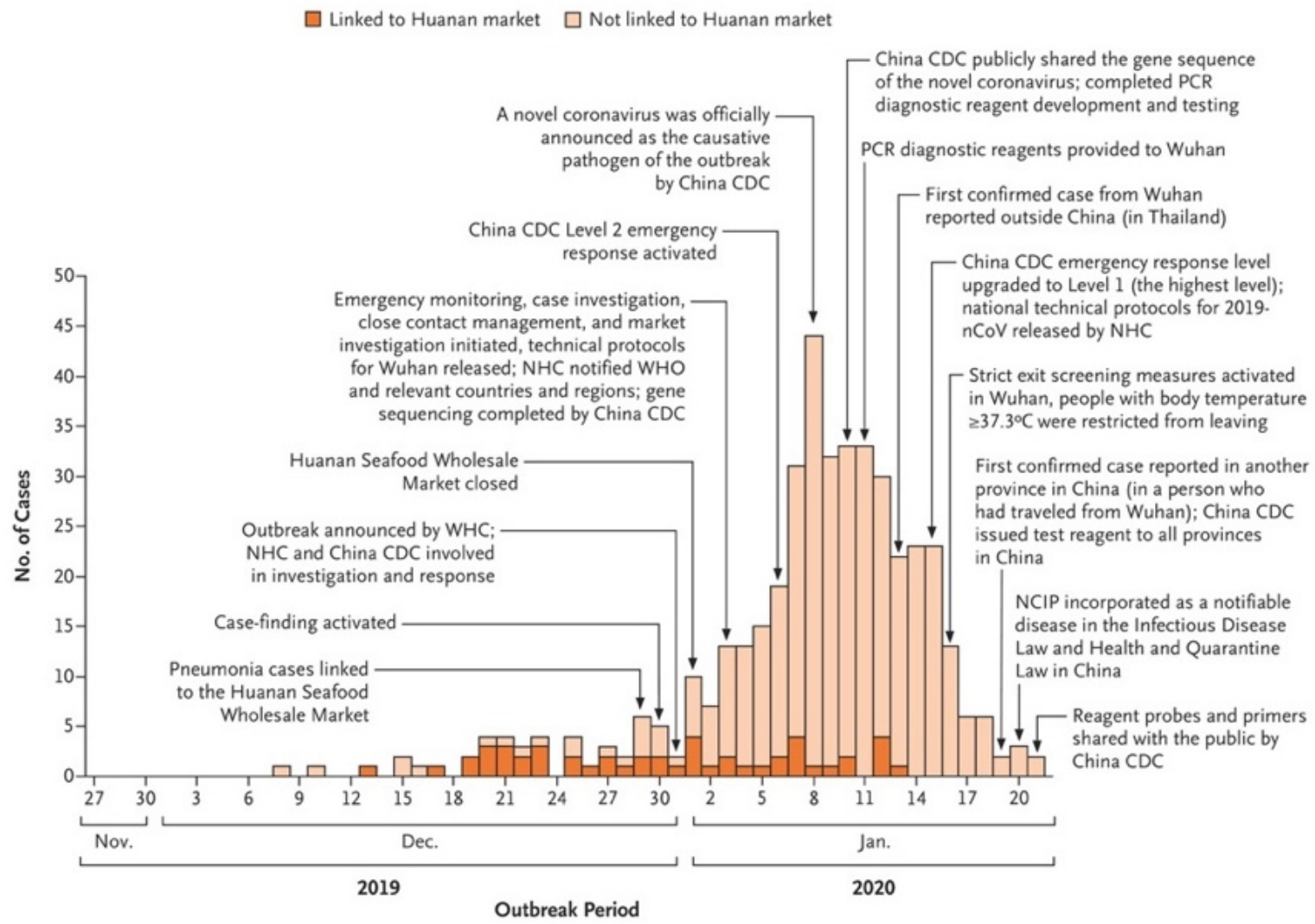

Figure 4. The onset of Illness among the First 425 Confirmed Cases of Novel Coronavirus (2019-nCoV)-Infected Pneumonia (NCIP) in Wuhan, China [25].

mode, if Wuhan did not implement lockdown and other measures, then by February 19, the number of confirmed cases of COVID-19 outside Wuhan will reach 744,000 $( \pm 156,000)$ [28]. Since the Chinese government intervened in the Wuhan and implemented the lockdown and other measures, the actual number of cases was reduced by $96 \%$ compared to the case without any intervention [28].

\subsection{Features in Dalian and Shenyang}

Because of effective control, China was stable in the early stage of the epidemic. However, some of the cases of COVID-19 are still reported every day. In some cities, there may suddenly appear small outbreaks - for example, Dalian and Shenyang.

Dalian is a city located on the North Pacific coast of China, and it is one of the top pots in China. In Dalian, it needs to handle about 10 million TEUs each year [29]. However, due to an epidemic, the cold chain portion of the port in Dalian port was forcibly closed. On December 15,2020 , Dalian reported four new cases of asymptomatic infection with COVID-19. The government quickly developed an investigation of the source of infection. After epidemiological investigations, nucleic acid testing, serum antibody testing, and other kinds of analysis were conducted on personnel, goods, and environmental specimens to discover the source of the epidemic and control the chain of transmission. This infection is about Dalian Yidu Port's cold chain because the products from the cold chain detected COVID-19. The on-site epidemiological investigation and molecular epidemiological analysis concluded that the beginning of epidemic infection was five dock workers [30]. As of January 2, 2021, Dalian has reported 78 cases of COVID19 and 30 cases of asymptomatic infections [30].

After the outbreak in Dalian, some of the departments, such as the Control mechanism of the Chinese Council and the National Health Commission, attached great importance to it. On December 22, a research group was 
assigned to Dalian to guide and assist in investigating the epidemic. The group comprises ten experts in the fields of medical management, epidemiology, virology, disease prevention and control, and health emergency. During the outbreak period, some areas in Dalian had been required to be blocked. Schools and other public places have also been closed. Residents may ask to stay at home as much as possible except working. At that time, the government also launched a large-scale test program to determine the scope of the city. On December 25, millions of residents in six districts of the town were required to be tested immediately [29]. The government also began to analyze cases and virus samples and found that they were different from previous virus samples in Dalian. After continuous investigation, the virus had the same characteristics as the virus in the European family of the L-genotype. The final

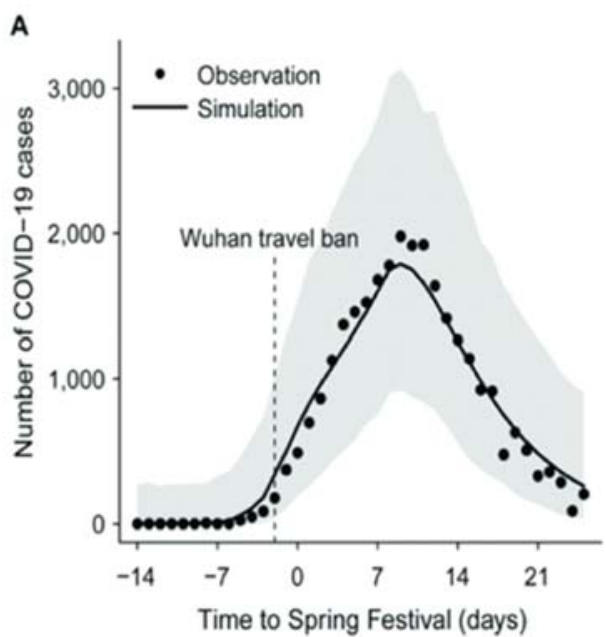

results supported that the source of transmission was coming from abroad.

On December 23, 2020, Shenyang confirmed a new case of COVID-19. The infected person is a woman who returned from abroad on November 29, 2020. According to China's new policy for the people who back from other countries, they must take 14 days of isolation in the place that the government arranged. During the isolation period, they must accept observation and nuclear acid tests. However, the result of the nucleic acid tests and serum antibody test of this infected person was negative. After the end of the isolation on December 13, that woman was detected to be infected with the COVID-19 on December 23 [31].

Since the patients have been active in different public places within ten days, the government attaches

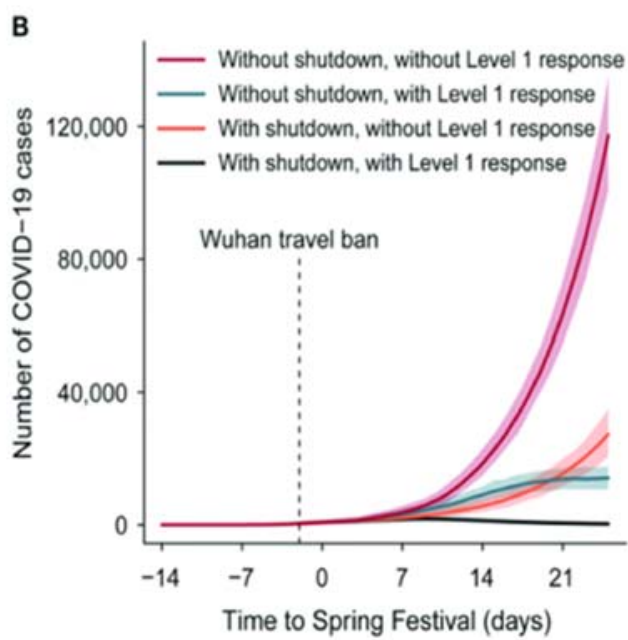

Figure 5. (A) Epidemic model of daily report of confirmed cases for 31 provinces. Hubei province does not include Wuhan. (B). Without Wuhan shutdown, and with (blue) or without (red) interventions implemented as part of the level 1 national emergency response, with Wuhan shutdown and with (black) or without (orange) the intervention. The dashed lines in (A) and (B) represent the date of the Wuhan shutdown and the start of the emergency response on January 23. The regions with shadows in (A) and (B) determine the $95 \%$ prediction envelopes [27].

great importance to this and then came out with various measures. The first measure is to treat patients actively. The city's hospital initiates the medical treatment plan, organizes a team of experts, formulates treatment plans, and spares no effort to treat patients. The second measure is to investigate the contacts thoroughly. If the contacts are found, they will be immediately transferred for centralized isolation and accept the medical observation and nucleic acid testing. The third measure is to delineate the epidemic point scientifically. According to the patient's activity trajectory and scope, the professionals will plan and disinfect, thus it can cut off the transmission route and eliminate the risk of transmission [31].

\subsection{Shijiazhuang}

Shijiazhuang is located in Hebei Province and is the capital of Hebei province. The outbreak in Shijiazhuang was without warning. According to the situation at that time, there were fifty-one new confirmed cases of COVID-19 in Hebei Province, and 50 cases were reported in Shijiazhuang on January 6, 2021 [32]. After the outbreak started in Shijiazhuang, the entire city declared a state of emergency and implemented lockdown in severe areas. All kinds of public transportation, such as highspeed rail, and airplanes had all been suspended. The most severely affected area is the Gaocheng district of Shijiazhuang, which was classified as a high-risk area. As of January 8, a total of 259 positive cases and 147 asymptomatic cases were detected in this area [33]. After the outbreak, the Shijiazhuang government established an emergency team for responding to the sudden outbreak. On January 6, Shijiazhuang initiated a number of measures and began a plan for a three-day full-time nucleic acid test for all citizens [32]. From the early morning of the 6 th to the end of the test at noon on the 9th, a total of $10,251,875$ people were tested [32]. This measure effectively blocking the spread of the epidemic and control the outbreak.

This epidemic is defined as super-spreading since it infected a lot of people. The reason for this epidemic in Shijiazhuang was few people attended a wedding and felt uncomfortable. Still, they did not go to the hospital and then caused a greater spread of the virus. This superspread may also relate to the weather. Dongyan Jin, a virus expert at the University of Hong Kong, mentioned that it 
is more likely to cause the spread of the epidemic if the people are indoors in severe winter. At that time, Shijiazhuang was in January, and the cold weather may be one of the factors that cause the transmission [34].

\section{Referential experiences from the pandemic}

Since December 2019, the COVID-19 had widely spread in China and the whole world, which featured strong diffusion power and high mortality. There was no specifically effective drug or vaccine, so the disease brought much threat to public health and life security. At present, the unified criteria of strategy for public health emergency have not been introduced yet, so it's significant to summarize and formulate feasible clinical measures to face more challenges and better protect the safety and basic authority of patients.

Public health emergency means the event which happens abruptly and possibly leads to severe damage to public health, including major infectious disease, mass disease of unknown cause, major food, and occupational poisoning and so on. It refers to the crowd disease involving a lot of provinces with evidence diffusion trend.

\subsection{Strengthen prevention and control measures of nosocomial infection}

In principle, perinatal pregnant women who are diagnosed or suspected of a pandemic should give birth in the corresponding designated hospitals. A team composed of multidisciplinary members is responsible for managing the isolation ward. Members should include obstetricians, neonatologists, infection doctors, and respiratory medicine, doctors, intensive care medicine doctors, anesthesiologists, nurses, etc. All present personnel needs to be equipped with third-level protection, a full set of protective equipment (including hats, goggles, protective masks, N95 masks, shoe covers, protective clothing). And all personnel shall plan the route in advance and conduct strict terminal disinfection of the goods, vehicles, and passages after the transportation. On-site treatment principally requires an inter-hospital transfer, and it shall be carried out in accordance with the requirements of inter-hospital transfer.

In outpatient triage, access to the outpatient clinic should be restricted. Measure the body temperature at the entrance of the outpatient clinic and ask about the aforementioned epidemiological contact history. If necessary, the patient must go to the specific clinic, and the expert group will determine whether it is a suspected case. Patients are admitted to the suspected isolation ward after complete check and inspection.

Both suspected and confirmed infected patients need to be isolated. Set up suspected infection wards and confirmed infection wards. It is best to isolate them inwards with negative pressure conditions. If there is no negative pressure ward, at least isolate in single rooms with independent air circulation systems. Dedicated passage, away from ordinary wards, strictly separate the office area (clean area), buffer zone, and isolation area, set up eye-catching signs at the door, and family members are prohibited from entering the isolation area for visits. The following measures shall be taken during observation and treatment:

All personnel entering the isolation ward shall be equipped with a full set of protective equipment and strengthen protection or even strict protection shall be adopted based on standard protection; to reduce the risk, the pregnant mother should avoid breastfeeding before the infection is cured or eliminated. The medical waste generated by the child is placed in a double-layer infectious medical waste bag, sealed in layers, marked on the sealing label, and handed over separately. Nondisposable medical fabrics used by patients should be collected at the bedside [35-38].

\subsection{Improve psychological construction}

The pandemic outbreak brings great pressure to families and medical staff. Medical staff may be psychologically affected by long-term overload emergency preparations and work, lack of medical resources, and the outcome of the cure.

During the epidemic, medical staff should receive psychological counseling, and self-care of medical staff is very important to maintain the strength of hospitals. So, the psychological consultation working group should provide psychological support to the medical staff on site. Carry out mental health assessment of front-line medical staff, strengthen psychological assistance measures, and achieve early detection, early intervention, and early guidance of psychological problems. Open a green channel for the families of frontline medical staff to seek medical treatment, establish a family system for community cadres to contact and help frontline medical staff, and give more help and care to the education of the children of frontline medical staff. At the same time, actively communicate with patients' family members through WeChat, telephone, etc., to relieve their tension [39].

When it comes to the vaccine or other necessary interventions, the government should get the willingness of participants with sufficient emergency use authorization (EUA). A survey was conducted to explain relationships between demographics and psychosocial predictors of intent to get a COVID-19 vaccine as well as willingness under EUA. Two measures the survey mainly took were the Health Belief Model (HBM) and the Theory of Planned Behavior constructs (TPB). It showed that individuals were less likely to get a future vaccine under EUA, thus, researchers further probed the impact of barriers. Predictions of lower intent included: high scores on cannot be bothered to get the vaccine, fear of needles, concern about side effects, possible vaccine shortage, and concern about rushed development of the vaccine. As noted by Jamison et al, trust in public institutions "may take considerable time and effort to repair." Efforts may need to go beyond just communications campaigns correcting misinformation about emergency interventions to also focus on re-establishing public trust in government agencies [40]. 


\subsection{Increase administration and management in fundamental systems}

So far, the effect of administration and management in different industries has been irregular for a long time. Those industries with evident economic benefits like administration, finance, education, and medicine are all under systematic management, while the foodservice industry and underlying services are lack control. According to the investigation, more than 10 hygiene and food safety incidents occurred in China in recent years. As to the origination of COVID-19, which was also resulted from overeating wildlife and the failure of the food regulatory agency, but human factors are not excluded. Therefore, the fundamental systems gradually become the core of the circle of health business. Here the government should devote more to increasing administration and management in fundamental systems, such as updating the status of each type of farmers' markets and strictly controlling the wholesale quality of products $[41,42]$.

\section{Conclusion}

This paper began with a general description of the COVID-19 epidemic in China (confirmed cases, deaths, fatality rate, etc.). According to the current prevention and control of COVID-19 in China, we can see that the epidemic in the Chinese mainland has been temporarily controlled, but there is still a risk of rebound. The clinical classification of 2019-COVID can be divided into four types: light, general, heavy, and dangerous in China, patients of different levels will be given corresponding treatment regimens.

After introducing the overall situation, this paper has carried on a detailed description for some local areas with characteristics, including the source of infection, the transmission, and some local government policies.

This paper summarized the source of the COVID-19 outbreak (the South China Seafood Market), the features of the virus (Beta-coronavirus), and the routes of transmission (4 routes) in Wuhan. At the same time, we discussed some new sources and transmission routes of new local cases in some areas after the basic prevention and control of the epidemic. We listed Dalian's imported epidemic, Shenyang's quarantined transmission, and Shijiazhuang's village transmission as reference areas. By comparing the former and the latter three, the article concluded the different trends caused by the transmission modes of novel coronavirus.

This paper also argued when different cities suffered from COVID-19 in different periods. The government would consistently implement various measures to control the outbreak and cut the transmission of the COVID virus. Cities in this paper include Wuhan, Dalian, Shenyang, Shijiazhuang. The epidemics in these places are symbolic and have special significance. Compare to these four cities, we identify the main reason for causing outbreaks in each city. Our finding shows that according to the reasons for causing outbreaks in each city, the Chinese government implemented different measures to control and solve the problem. It shows the response of the Chinese government was rapid and effective.

We have learned a lot from the COVID-19, and to better face the next potential pandemic, it's primary for us to strengthen prevention and control measures of nosocomial infection, improve psychological construction and increase administration and management in fundamental systems. However, there is still a long way we need to go to prevent and overcome such outbreaks.

\section{References}

1. Zhang LH, Li BH, Jia P, et al. SARS-CoV-2, Analysis of global research status [J]. Journal of biomedical engineering, 2020,37(02):236-249.

2. Wang M, Zhang AH, Shen Q, et al. How to prevent novel coronavirus infection in children's blood purificati-on center $[\mathrm{J}]$. Journal of clinical kidney disease, 2020,20(2): 1-9.

3. Zhao WM, Song SH, Chen ML, et al. The 2019 novel coronavirus resource [J]. Yi Chuan, 2020, 42(2): 212221.

4. National Health Commission of the people's Republic of China. Novel coronavirus infection pneumonia is included in the management of notifiable infectious diseases. [EB/OL] (2020-01-21) [2021-01-27]. http://www.nhc.gov.cn/jkj/s 7916/202001/44a3b8245e8049d2837a4f27529cd386 .shtml.

5. Lipsitch M, Cohen T, Cooper B, et al. Transmission dynamics and control of severe acute respiratory syndro-me [J]. Science, 2003, 300(5627): 1966-1970.

6. Special Expert Group for Control of the Epidemic of Novel Coronavirus Pneumonia of the Chinese. An update on the epidemiological characteristics of novel coronavirus pneumonia (COVID-19) [J]. Chin J Viral Dis, 2020, 10(02):86-92.

7. Baidu. Report on novel coronavirus pneumonia epidemic in real time. [EB/OL].(2020-07-31)[202101-27].https://voi-

ce.baidu.com/act/newpneumonia/newpn eumonia/?from=osari_pc_3\#tab0.

8. General Office of the State Health Commission, office of the State Administration of traditional Chinese medicine notification of New Coronavirus pneumonia diagnosis and treatment plan (trial version 7) $[\mathrm{EB} / \mathrm{OL}] .[2020.03 .04]$. http: / /www.nhc.gov . cn /yzygj /s7653p /202003 /46c9294a7dfe4cef80dc7f5912eb1989.shtml.

9. YUAN R, XIN Q Q, TANG S H, et al. Treatment of COVID-19 guided by holistic view of traditional Chinese medicine-therapy aimed at both viral and host[J]. China Journal of Chinese Materia Medica, 2020, 45(7): 1521-1525.

10. Wang Qian. Practice of integrated traditional Chinese and Western medicine in the treatment of mild pneumonia in New Coronavirus [J]. Tianjin Chinese medicine, 2021,38 (02): 171-175. 
11. Zou Lu, Yu Xiao, Wu Yuqin, sun Ding, Wu Yingen, Fang Hong. Analysis and Reflection on prevention and treatment of severe New Coronavirus pneumonia by Chinese medicine [J]. Journal of Shanghai University of Traditional Chinese Medicine, 2020,34 (02): 11-16.

12. PEDERSEN S F, HO Y C. SARS-CoV-2: a storm is raging. $[\mathrm{J}]$. J Clin Invest, 2020: 137647. DOI: 10.1172 /JCI137647.

13. Huang C, Wang Y, Li X, Ren L, Zhao J, Hu Y, Zhang L, Fan G, Xu J, Gu X, Cheng Z, Yu T, Xia J, Wei Y, Wu W, Xie X, Yin W, Li H, Liu M, Xiao Y, Gao H, Guo L, Xie J, Wang G, Jiang R, Gao Z, Jin Q, Wang J, Cao B. Clinical features of patients infected with 2019 novel coronavirus in Wuhan, China. Lancet. 2020 Feb 15;395(10223):497-506. doi: 10.1016/S0140-6736(20)30183-5. Epub 2020 Jan 24. Erratum in: Lancet. 2020 Jan 30;: PMID: 31986264; PMCID: PMC7159299.

14. Information from https://ncov.dxy.cn/ncovh5/view/pneumonia_area?ai $\mathrm{d}=420000 \&$ from $=\mathrm{dxy} \&$ link $=\&$ share $=0 \&$ source $=$ app share \&entrysource $=$ APPiOS \&entrysource $=$ APPiOS

15. Information from http://wjw.wuhan.gov.cn/gsgg/202004/t20200430_1 199576.shtml

16. Xu X, Chen P, Wang J, Feng J, Zhou H, Li X, Zhong W, Hao P. Evolution of the novel coronavirus from the ongoing Wuhan outbreak and modeling of its spike protein for risk of human transmission. Sci China Life Sci. 2020 Mar;63(3):457-460. doi: 10.1007/s11427-020-1637-5. Epub 2020 Jan 21. PMID: 32009228; PMCID: PMC7089049.

17. Sun Chen, Jiang Yanan, Zhao Jimin. [12] Wang Y, Wang Y, Wang Y, et al. The clinical significance of COVID-19 in the treatment of patients with acute coronary heart disease [J]. J Clin Microbiol, 2015, 35 (6): 1085-1092.

18. National Health Commission. COVID - 19 medical 6 (trial edition) [EB/OL]. (2020-02-19)[2020-0302].HTTP://http://www.nhc.gov.cn/yzygj/s7653p/20 2002/8334a8326dd94d329df351d7da 8aefc2.shtml.

19. Information from https:/www.maritimeexecutive.com/article/china-s-dalian-port-cityresponding-to-new-covid-19-outbreak

20. Information from https://news.sina.com.cn/c/202101-03/doc-iiznctke9946124.shtml

21. Information from http://wjw.shenyang.gov.cn/html/WSJ/16111943375 4444/152574818961422/152574818961422/337544 4426631593.html

22. Information

from http://wjw.shenyang.gov.cn/html/WSJ/16111943375 4444/152574818961422/152574818961422/337544 4481949243.html

23. Information from https://news.sina.com.cn/c/202101-11/doc-iiznezxt1875523.shtml\#
24. Information from http://www.chinacdc.cn/jkzt/crb/zl/szkb_11803/jszl_ 11809/202002/t20200202 212142.html

25. Information from https://www.nejm.org/doi/full/10.1056/NEJMoa200 1316

26. Information from https://weileizeng.github.io/wuhan2020-timeline/

27. ht Tian, H., Liu, Y., Li, Y., Wu, C., Chen, B., Kraemer, M., . . . Dye, C. (2020, May 08). An investigation of transmission control measures during the first 50 days of the COVID-19 epidemic in China. Retrieved April 16, 2021, from https://science.sciencemag.org/content/368/6491/63 8

28. Information from http://www.chinacdc.cn/gwxx/202004/t20200407_2 15838.html

29. Information from https://www.maritimeexecutive.com/article/china-s-dalian-port-cityresponding-to-new-covid-19-outbreak

30. Information from https://www.medsci.cn/article/show_article.do?id=9 ca4205294d5

31. Information from http://wjw.shenyang.gov.cn/html/WSJ/16111943375 4444/152574818961422/152574818961422/337544 4426631593.html

32. Information from http://www.sjz.gov.cn/col/1598783318372/2021/01/ 10/1610241568925.html

33. Information from https://www.dw.com/zh /a56165322

34. Information from https://www.voachinese.com/a/virus-restrictionsheightened-China-province-20210107/5728537.html

35. Medical Association of Chinese People's Liberation Army et al. Zhongguo dang dai er ke za zhi $=$ Chinese journal of contemporary pediatrics vol. 22,3 (2020): 205-210. doi:10.7499/j.issn.1008-8830.2020.03.005

36. Atzrodt CL, Maknojia I, McCarthy RDP, et al. A Guide to COVID-19: a global pandemic caused by the novel coronavirus SARS-CoV-2. FEBS J. 2020;287(17):3633-3650. doi:10.1111/febs.15375

37. Triggle CR, Bansal D, Farag EABA, Ding H, Sultan AA. COVID-19: Learning from Lessons To Guide Treatment and Prevention Interventions. mSphere. 2020;5(3):e00317-20. Published 2020 May 13. doi:10.1128/mSphere.00317-20

38. Shen F, Min C, Lu Y, Chu Y. The effect of cognition and affect on preventive behaviors during the COVID-19 pandemic: a cross-sectional study in China. BMC Public Health. 2021;21(1):722. Published 2021 Apr 14. doi:10.1186/s12889-02110784-y

39. Li H, Liu Z, Ge J. Scientific research progress of COVID-19/SARS-CoV-2 in the first five months. J Cell Mol Med. 2020 Jun;24(12):6558-6570. doi: 
10.1111/jcmm.15364. Epub 2020 May 7. PMID: 32320516 ; PMCID: PMC7264656.

40. Guidry JPD, Laestadius LI, Vraga EK, et al. Willingness to get the COVID-19 vaccine with and without emergency use authorization. Am J Infect Control. 2021;49(2):137-142. doi:10.1016/j.ajic.2020.11.018

41. Lupieri S. Refugee Health During the Covid-19 Pandemic: A Review of Global Policy Responses. Risk Manag Healthc Policy. 2021;14:1373-1378. Published 2021 Apr 6. doi:10.2147/RMHP.S259680

42. Singh D, Yi SV. On the origin and evolution of SARS-CoV-2 [published online ahead of print, 2021 Apr 16]. Exp Mol Med. 2021;1-11. doi:10.1038/s12276-021-00604-Z 